\title{
Wired for Society: Cognizing Pathways to Society and Culture
}

\author{
Laurence Kaufmann • Fabrice Clément
}

Published online: 28 January 2014

(C) Springer Science+Business Media Dordrecht 2014

\begin{abstract}
While cognitive scientists increase their tentative incursions in the social domains traditionally reserved for social scientists, most sociologists and anthropologists keep decrying those attempts as reductionist or, at least, irrelevant. In this paper, we argue that collaboration between social and cognitive sciences is necessary to understand the impact of the social environment on the shaping of our mind. More specifically, we dwell on the cognitive strategies and early-developing deontic expectations, termed naïve sociology, which enable well-adapted individuals to constitute, maintain and understand basic social relationships. In order to specify the way in which the demanding character of typical social relationships can be recognized in situ, we introduce the concept of "deontic affordances". Finally, we shed light on the continuum that might relate a primitive naïve sociology, dedicated to the processing of invariant properties of the social life and a mature naïve sociology, necessary for dealing with the variable properties of cultural forms of life.
\end{abstract}

Keywords Cognitive science $\cdot$ Naïve sociology $\cdot$ Deontic affordances $\cdot$ Social relationships

\footnotetext{
L. Kaufmann $(\bowtie)$

Laboratory of Sociology, Institute of Social Sciences, University

of Lausanne, 1015 Dorigny, Switzerland

e-mail: Laurence.Kaufmann@unil.ch

F. Clément

Cognitive Science Center, University of Neuchâtel,

2000 Neuchâtel, Switzerland

e-mail: Fabrice.Clement@unine.ch
}

\section{Introduction: Fear of nature}

From the perspective of the history of the philosophy of sciences, the collective resistance that most social scientists oppose to the so-called "cognitive revolution" is striking. They deliberately ignore, if not reject, the considerable body of knowledge with respect to human nature that cognitive and developmental psychology, neuropsychology, neurosciences and evolutionary theory have accumulated during the last decades. The origin of this indifference can be summed up by Dilthey's founding distinction between the natural sciences (Naturwissenschaften), built upon the discoveries of explanatory physical mechanisms, and the human sciences (Geisteswissenschaften), driven by the hermeneutic comprehension of history and culture as the "objectifications" of the human mind (Dilthey 1883; Havelange 1998). From this hermeneutical standpoint, cultural objectifications are assumed to have their own ontology, in this case a "historical ontology," based upon the shared meanings and impersonal rules that constitute the objective mind of a given community (Hacking 1999). This objective mind would lie by definition outside, above, beyond or between the individual minds, in the public realm of common practices, norms of conduct and religious, juridical and philosophical representations that make intelligible any mental and physical activities (Descombes 1996). In short, for most social scientists, the explanation of human action cannot be grounded in the first nature of the mind, that is, in the universal mechanisms of information processing that cognitive science focuses on (Winch 1958; Durkheim 1912). Sociological explanations of action are said to be necessarily based upon the second nature of the mind, that is, the more or less collective principles of acting that furnish it (Winch 1958; Durkheim 1912; Weber [1904] 1949; Bourdieu 1979; Garfinkel 1967). Even opposite 
sociological paradigms such as methodological individualism and holism tend to agree that the intentional order of culture must be strongly distinguished from the natural order of material causes and cerebral mechanisms. ${ }^{1}$ Such distinction, as well as the fear of reductionism associated with biology, has led social scientists to even see "social relations as a denial of nature" (Hirst and Wooley 1982: 22). ${ }^{2}$

The problem with these dualist views of the relationship between nature and culture, instinct and sociality, is that they prevent the development of an "integrated model of the study of the human" (Schaeffer 2007). For it is precisely the gap between the human and the animal, cultural history and biological evolution, that naturalism aims to fill in. Naturalism aims to harmonize, in the sense of "making compatible", research done in social science with knowledge accumulated in the natural sciences. Interestingly, the project of harmonizing social and natural sciences seems less and less far-fetched thanks to the "social scientistcompatible" view of cognition that the cognitive sciences are currently developing. Actually, the study of cognition is no longer monopolized by the functionalist model of artificial intelligence that used to depict, in the 1980s, a "culture-proof" cognition, driven by low-level mechanisms impervious to cultural framing or supra-individual categorization. On the contrary, a growing body of research in social neuroscience and evolutionary psychology demonstrates the impact of the social environment on the shaping of our brain. The "Social Brain Hypothesis", in particular, posits that cognitive abilities of human and nonhuman primates are the result of a long process of selection where enduring environmental constraints played a crucial role, including social constraints such as social relationships and coalition building (Humphrey 1976; Dunbar 2003). A more human-specific hypothesis, called the "Cultural Intelligence Hypothesis", suggests that the human mind might even be "prewired" for cultural learning and knowledge acquisition, particularly that of conventional symbols, complex tools, and institutional

\footnotetext{
${ }^{1}$ Of course, the argument of the individualist approaches to the social, for which the state of society ultimately depends on firstperson conscious, reflexive decisions of individuals, is very different from the holistic argument according to which there is an irreducible social reality, both external and constraining (Durkheim 1912). For individualist sociologists, the problem is that cognitive science unduly dismantles the rational actor and replaces it with an organism, conceived as the infra-individual site of the production, conflict and coalition of mostly unconscious cognitive representations and micromechanisms. In this case, the mistake of the cognitive sciences is not to neglect the weight of the social but to weaken "the empire of the will" proper to the individual-as-rational-self (Bronner 2006).

2 The recent, lively French debate that the social naturalism proposed by Kaufmann and Cordonier (2011) has prompted among sociologists shows the persistence of this view.
}

rules (Tomasello 1999a, b; Herrmann et al. 2007). Despite these promising, interdisciplinary lines of reflection on our cognitive inclinations to society and culture, social scientists tend to treat them as irrelevant (Quéré 2011; Ogien 2011).

This isolationist trend can be overcome only if a fruitful dialogue between social science and cognitive science is established. To contribute to this dialogue, this paper draws from research in developmental, comparative, and evolutionary psychology in order to shed light on the invariant cognitive commonalities that enable social beings to hold society together. For the social sciences, the fine-grained account of such cognitive commonalities is of a particular interest: it enables to open the "black box" of the mysterious process of socialization whereby individuals are supposed to accommodate and assimilate the beliefs, norms and values of their community.

The paper is structured as follows. In the first part, we will sum up the main arguments in favor of the domainspecific organization of our cognitive architecture. After emphasizing the key role of the social environment in our natural history, we will dwell on the domain-specific inferential strategies and early-developing deontic expectations, mostly shared with non-human primates, which enable well-adapted individuals to cope with their social surroundings. Following in part Lawrence Hirschfeld's proposal $(1999,2001)$, we will call this set of capacities "naïve sociology". Unlike Hirschfeld, however, we will not defend a view of "naïve sociology" as a naïve social psychology, essentially focused on group membership processing. We will propose a view of naïve sociology as a more general mode of social relationships processing that enables human and non-human primates to detect typical kinds of interactions (inter-actions) and to use them in order to determine how to behave toward others and what to expect from them. In the second part of our paper, we will specify the way social beings recognize the demanding character of the typical relationships they are confronted with thanks to the concept of "deontic affordances". As will be argued, deontic affordances that enable social perceivers to fore-see what will or should happen next are the perceptual basis of the deontic inferences that are at the heart of our relational account of naïve sociology. The third part of our paper outlines some lines of continuity between the primitive naïve sociology dedicated to the invariant properties of the social life and a mature naïve sociology, necessary for dealing with the variable properties of cultural forms of life. By way of conclusion, we will argue that research on the prewired set of categorizations and expectations that enable and constrain the way society can come to the human mind can be fruitful for both cognitive and social science. Not only such research would prompt social scientists to be, at last, realistic about the mental but 
it would also prompt cognitive scientists to be more realistic about the relational and deontic nature of social life.

\section{The Cognitive Processing of the Social Domain}

It is nowadays widely admitted that the forces of evolution, and in particular the process of selection, were not exerted only on our bodies. The minds of evolved organisms, just like their physiology, are also genetically adapted to their ancestral environment. At the heart of the cognitive equipment that complex organisms have evolved is the capacity to detect particular sorts of relevant information and to use them to anticipate future events. As the philosopher Daniel Dennett put it, "a mind is fundamentally an anticipator, an expectation generator" (Dennett 1996: 57).

The recent burgeoning of research blending evolution with psychology has given rise to interesting hypotheses about the way organisms have internalized, into their cognitive architecture, the structural regularities of their physical and social environment. Many authors have thus argued that natural selection gives priority to domain-specific information and learning processing over domaingeneral mechanisms, too costly in time and energy (Cosmides and Tooby 1994; Sperber et al. 1995; Herrmann et al. 2007). Domain-specific data processing ensures an "informational match" between the cognitive skills of a given organism and the problems raised by its environment, increasing thereby its fitness (Gelman and Williams 1998). At the very least, most cognitive scientists agree that evolved organisms are inclined to process different kinds of incoming information in specific ways.

If a degree of domain-specific information processing is not called into question within cognitive science, the nature, scope and number of those domain-specific mechanisms are more controversial. Many domain-specific information-processing systems have been proposed, duly organized as a function of the properties of the different kinds of entities that they pick out and the core inferential principles that support reasoning about them: naïve physics (physical entities-causal law) (Baillargeon 1987; Spelke 1994), naïve biology (living beings-genetic transmission) (Atran 1998; Keil 1998), naïve psychology (mental statesintentional attribution) (Wellman 1990; Baron-Cohen et al. 2000), naïve morality (rules of welfare and justice-ethical evaluation) (Turiel 1983; Nucci 2001), and naïve arithmetic (small numbers-quantificational computation) (Dehaene et al. 1999; Baillargeon and Carey 2012). Those different domains of information processing have been revealed in part by developmental psychology, which shows how children's knowledge about objects, people and events expands very rapidly from the very first months of infancy, including causal expectations that are underdetermined by experience and impervious to counter-evidence (Hirschfeld and Gelman 1994). Domain-specific information processing has also been revealed by research in psychopathology, suggesting for instance that the developmental disorder of people with autism is characterized by the domain-specific impairment of the "mentalizing mechanism" necessary to work out what is going on inside other people's heads (Baron-Cohen et al. 2000; Leslie 1991).

Given the importance of the social environment for humans, the fact that "naïve sociology" has not been granted much attention compared to the other domainspecific mechanisms is somewhat surprising. With a few notable exceptions (Hirschfeld 1995, 1999, 2001; Jackendoff 1994, 1999; Cummins 1999), social entities such as social rules, hierarchies, or groups have rarely been taken into account as a particular, specific domain of cognition. One likely reason for this relative indifference is that the "social" domain focused on by most cognitive scientists is essentially intersubjective and can be processed, as such, by naïve psychology. In intersubjective interactions, indeed, the mental states of others, whether they be intentions, knowledge, beliefs, or desires, are particularly relevant. As Harris (2006) points out, activities of conversation feed on the discrepancies in perspective and belief and on the exchange of viewpoints differences. The intersubjective framing of the social domain has thus led cognitive scientists to account for social cognition in mentalistic terms. In developmental and comparative psychology, as well as in psychopathology and social neuroscience, the ability to read the minds and thoughts of conspecific - the so-called Theory of Mind - is said to be the primary, pervasive way of interacting appropriately with others (Astington 2004; Spaulding 2010). This extensive view of mentalizing as the essential way of navigating the social world is precisely what the hypothesis of "naïve sociology" as another foundational domain of social cognition aims to call into question.

\subsection{Revisiting Social Cognition}

The mentalistic cast of social cognition within cognitive science has been challenged by the anthropologist Lawrence Hirschfeld, who proposed the existence of a specific social faculty that he coined "naïve sociology" (Hirschfeld 1995, 1999, 2006). For him, this faculty refers to the capacity to rapidly detect affiliations and memberships, and to reason in terms of discrete "human kinds", that is, groups and social categories like race, gender, caste, kinship or social occupations (Hirschfeld 2001). For instance, when confronted with someone who violates the expectations linked to a given situation (e.g., driving on the 
freeway), people do not seek out the possible beliefs of the driver causing the violation. Rather, they draw on assumptions and stereotypes about the driver's social position (e.g., women drivers who do not master the requisite skills, Republican drivers in their big Hummer who do not believe that they are bound by traffic rules, etc.) (Hirschfeld 2006). Ultimately grounded in the categorical thinking that social psychology emphasizes, naïve sociology would thus be dedicated to the processing of the grouplevel, socially relevant traits that allow social perceivers to identify what kind of people they are dealing with and to take such group-level identification as a basis for inference, prediction and action (Hirschfeld 1999; Hirschfeld and Gelman 1994).

Different lines of research confirm Hirschfeld's emphasis on the cognitive strategies that allow adults and children to process group membership, allegiance and social coalitions. For instance, Social Exchange Theory advocated by Cosmides et al. (2003) assumes the existence of a mandatory but flexible capacity for reasoning about shifting alliances and social exchanges, mainly thanks to coalitional categorization, expected reciprocation and cheater detection (Kurzban et al. 2001; Cosmides et al. 2005). In a new, fast developing domain of research, several developmental psychologists reveal children's proclivities for parsing the social world into groups. Indeed preschoolers show abstract expectations about how group members relate to one another and use social categories as an inductively powerful means of predicting other's behavior (Diesendruck and Eldror 2011; Kurzban et al. 2001; Rhodes 2012; Shutts et al. 2011). In particular, preschoolers have a strong preference for the individuals who are similar to themselves, showing thereby a precocious emerging of implicit preferences for in-groups and hence an early division between "us" and "them" (Dunham et al. 2008 Kinzler and Spelke 2011). Also drawing from the rich and longstanding literature in social psychology, Spelke and Kinzler 2007 suggest that group membership might be a conceptual primitive. Apart from the four more established core systems "that stand at the foundation of our beliefs and values" (objects, agents, number and space), there would be a fifth core system. "Such fifth system serves to identify members of one's own social group in relation to members of other groups, and to guide social interactions with in- and out-group members" (Spelke and Kinzler 2007: 257).

Although group membership has certainly a key role to play in the non-mentalistic dimension of social cognition, one can wonder what exactly is the conceptual primitive at stake. Indeed, group membership is by definition a social relationship that restrains and enables the way group members relate and should relate to one another. From a theoretical point of view, seeing group membership as a type of primitive social relationship rather than a categorybased perception of persons has an important consequence. It enables to draw attention to other relational primitives that have been mostly left aside by developmental psychology, comparative psychology and cognitive anthropology: exchange, cooperation, but also competition and dominance. ${ }^{3}$ Our hypothesis is then along the same lines as "the relational models theory" that was forcefully proposed by Alain Fiske years ago on the basis of inter-cultural ethnographic fieldwork and experimental studies. To Fiske, humans have representations of foundational patterns of social relationships that enable them to generate social action and to make sense of others' social behavior, namely "authority ranking" (e.g., people attend to their positions in a linear ordering), "equality matching" (e.g., people keep track of the imbalances among them), "communal sharing" (e.g., people treat all members of a category as equivalent), and "market pricing" (e.g., people orient to ratio values) (Fiske 1992, 1994; Haslam and Fiske 2004). Compared to Fiske's relational theory, our naïve sociology hypothesis puts more emphasis on domain-specificity and leaves still open the range of rudimentary social relationships susceptible to be rapidly cognized, in particular by non human primates and young children. It is not sure, for instance, that market pricing, which seems more adjusted to a human and possibly adult way of reasoning, is mastered as early as the other relational patterns. But our overall hypothesis goes in the same direction as relational models theory: humans and possibly primates have representations of abstract types of social relations and ways of recognizing them in the social world. Those elementary types of relationships would be neither lexicalized, nor consciously cognized as such, but would contain universal features capable of specifying, both singly and in combination, outward forms of social relationships with minimal cognitive processing (Fiske and Fiske 2007; Jackendoff 2007). As Thomsen and Carey (2013) have recently emphasized, those core concepts of relationships would thus be part of a relatively small innate repertoire of abstract relational patterns that would enable us to recognize their realization in particular cultural relationships.

To illustrate this relational hypothesis, let us take the case of dominance, conceived here as an asymmetric dyadic relationship that establishes who has priority access to various physical or social resources (Hawley 1999; Watts 2010). A growing body of research suggests that the capacity of recognizing the relational pattern of dominance

\footnotetext{
${ }^{3}$ In guise of example, in the excellent, voluminous overview of child psychology recently published by Banaji and Gelman (2013), there are only two pages about dominance and it is not an entry in the index. To our knowledge, only two experimental studies have been published in developmental psychology on dominance processing, namely Mascaro and Csibra (2012) and Thomsen et al. (2011).
} 
and using it as a basis for inference and action is innate and partly shared with non-human species. Numerous studies indeed show that dominance cues are easily recognized and have a strong inductive potential in animal species. For instance, male bonnet macaques use dominance rank to assess which potential allies are likely to be effective in coalitions against their opponents (Silk 1999), Pinyon jays use transitive inference to predict social dominance (Paz-yMiño et al. 2004), and even fish gather status information from observing conspecifics interactions (Grosenick et al. 2007). Recent studies demonstrate that those recognitional and inferential capacities appear very precociously in human species. Infants as young as 15-month-old show a strong sensitivity to third-party asymmetric relationships that they expect to remain stable from one conflict (e.g., the dominant agent repeatedly pushes the subordinate so as to monopolize a given area) to another conflict (e.g., the two agents competing over a desired resource) (Mascaro and Csibra 2012). Other studies show that 9- and 10-month-old infants expect small agents to bow and prostrate in subordination to others of more formidable physical size (Thomsen et al. 2011). Recent developmental studies reveal that dominance expectations are cross-situational: 3 yearold children predict that a dominant who has imposed his choice on another will then exhibit higher competence in no-related games and will have more resources than the subordinate (Charafeddine et al. submitted). Such experimental demonstrations of early dominance processing confirm previous studies that have used natural observation and ethological analysis to reveal the sophisticated ways in which preschoolers use dominance to create political alignments and group coalitions (Strayer and Strayer 1976).

Taken together, these different studies suggest that dominance, even if it varies within and between species, from leadership-like assertiveness to hostile and aggressive control, has a "deep structure" that remains essentially the same (Hawley 1999). The fact that this deep relational structure is identified and used very early in ontogeny and phylogeny suggests that dominance processing might be an important part of the relationship-based naïve sociology that we hypothesize here. Of course, it is necessary to distinguish between a basic naïve sociology as a core system and its transformation, when it undergoes development, into a mature naïve sociology. While basic naïve sociology would enable to parse the elementary forms of dominance, also present in non-humans, mature naïve sociology would enable to process their context-relative significance as well as their more subtle and culturedependent variations, such as charisma or prestige. Although dominance or what Fiske and colleagues call "Authority Ranking" is available to all humans as a model for organizing their surroundings, cultures indeed differ in how positions in hierarchy are ascribed or achieved, enduring or transitory, and how they depend on coercion or, on the contrary, on personal merit, competency and ability (Fiske 2005; Haslam 2004).

\section{Fleshing Out "Naïve Sociology"}

While drawing significantly from Hirschfeld's view, our account of naïve sociology gives it a relational twist. Instead of being a kind of naïve social psychology that targets individuals as group members, the target domain of naïve sociology, we claim, would consist in elementary types of social relationships-including, of course, the relationship of affiliation (i.e., group membership).

Hypothesizing different types of social relationships as fundamental social units permits making an important distinction between naïve psychology and naïve sociology. The ontological primitive of both naïve psychology and naïve sociology understood as naïve social psychology is the person, whether as a seat of personal dispositions and attributes or as a seat of category-based stereotypes or group-level properties. By contrast, the ontological primitive of the naïve sociology hypothesized here is social relationships, or basic patterns of interaction. Although research is needed in developmental psychology and comparative psychology to specify those basic relational frames, one can assume without too much risk that they most likely include the following types of relations: group membership or affiliation as relation of inclusion (A affiliates oneself with a group $G$ ), dominance as an asymmetric relation ( $\mathrm{A}$ imposes her desire on $\mathrm{B}$ ), exchange as a symmetric, reciprocal relation (A gives $\mathrm{x}$ to $\mathrm{B}$ in return for $\mathrm{y}$ ), and possibly nurturance as a positive relation of dependency (A provides care and attention to B).

To sum up the argument that we have made so far, it seems that naïve sociology might well constitute a specific domain of its own. Just as the other domains are organized around a set of basic principles serving to identify the relevant entities of a given domain and to reason about these entities (Carey and Spelke 1996), naïve sociology is characterized by a coherent set of foundational concepts and core principles. Indeed, naïve sociology satisfies one of the main conditions of the functioning of our learning systems, namely the "conceptual parsing" of the world that Spelke (1994) speaks of. By operating the relational parsing of our social environment, naïve sociology enables us to see interactions as instantiations of competition, cooperation, or dominance and then others as allies or foes, superiors, inferiors or equals, givers or beneficiaries, and so on. Moreover, social relationships are governed by a core principle that might well be, as we will go on to argue, of a deontic nature. This core principle of social relationships is deontic because they are by definition patterned 
interactions: they allow social beings to expect that each time that the action A occurs, the re-action B should appropriately follow; and that whenever the action B occurs, it should have been preceded by the action A. For instance, the action of "giving an order" should be followed by the action of "obeying", the two sequences instantiating together a dominance relationship. The core principle that guides the way individuals reason about social relationships is deontic in the sense that it enables them to expect, in a relational-specific way, what should happen next. Thus affiliative relationships involve a deontic principle of mutual cooperation and assistance that raises normative expectations. These expectations appear surprisingly early in ontogeny; children as young as 26 months of age expect someone in distress to be helped by a "friend" rather than by a stranger (Beier et al. 2010).

The main systems of core knowledge such as knowledge of objects and their motions, or knowledge of agents and their goal-directed actions, have been proven to be present in humans but also in other animals, especially primates (Spelke et al. 2013). Given the ability of non-human primates to navigate third-party relationships, such as dominance, kinship, and group membership (Cheney and Seyfarth 2007), one can reasonably hypothesize that they have also at their disposal a naïve sociology. But such hypothesis is sustainable only if the deontic principle that we assume to be at the heart of social relationships monitoring is within the cognitive reach of young infants and non-human primates. Although studies on this topic are surprisingly scarce, recent research do provide evidence of elementary norms in other species. Thus chimpanzees show primitive expectations about the way others, for instance infants, should be treated. Far from being only based upon statistical regularities, those expectations have a deontic dimension, as revealed by the strong reactions elicited by their violation: conspecifics use third-party intervention and policing to reinforce the tolerance that adults are supposed to exhibit towards infants (Rudolf von Rohr et al. 2011). Other studies show that juvenile chimpanzees perceive prescriptive social rules concerning the intensity and noise expected when playing and use these to regulate their signaling behavior in play contexts (Flack et al. 2004). Deontic expectations in non-human primates are all the more likely as they do not necessarily involve the metarepresentational capacity to follow a norm or to have a theory of mind. Deontic expectations might rely on a more rudimentary cognitive mechanism, that is, the elementary sense of appropriateness of such and such behavior (e.g., protecting infants, obeying a dominant, etc.) (Andrews 2009). Such "sense of appropriateness" does not require the antecedent grasp of a norm determining which response is correct or incorrect; it can merely be a sense of "primitive normativity" already present, as such, in human infancy as well as in other non-human species (Ginsborg 2011; Sultanescu and Andrews 2013).

This being said, the way this minimal sense of normativity might appear in the eye of the beholder remains mostly obscure. Saying that the interactional norm of responding by action B to action A appears in the form of what is appropriate to do within a given type of relationship is far from sufficient. Concretely, how might the deontic force of social relationships be cognitively grasped without too complex cognitive processing? To start answering this question, we need to carefully distinguish, as the primatologist Robert Hinde (1976) suggested 40 years ago, two levels of social iteraction. The more abstract level is the level of relationships that we have emphasized until now, that is, the "patterning of interactions" resulting from the process of abstraction that both human and non human primates are capable of (Hinde 1976). Such "relationships mapping" allows individuals to specify what kind of action they are doing together (e.g., exchanging, dominating, cooperating, etc.). The less abstract level, Hinde says, is the level of the real-life, "concrete interactions" that are nothing but the instantiations of the abstract social relationships mentioned above: instances of exchanging (e.g., food-for-grooming), dominating (e.g., chasing a competitor), cooperating (e.g., caring for the kid of another female) and so on.

Once established the difference between these two levels of social relationships and cognitive abstraction, it is easier to better specify how the deontic force of situated interactions might be processed by individual minds. Our hypothesis is that interactions appear to social perceivers as a succession of affordances, each action affording a set of possible subsequent actions. In contrast with the standard affordance theory, we will contend that interactions do not only offer certain opportunities for action or reaction; they constrain the range of appropriate responses. This is precisely to account for this constraining character that we will use the concept of "deontic affordance", notably proposed by Carassa and Colombetti (2009). While remaining "cognitively parsimonious", the concept of "deontic affordance" provides a better understanding of how primitive normativity might be actually achieved and exploited in real interactions. This is what the next section aims to show.

\subsection{The Deontic Force of Social Affordances}

Initially, affordances have been conceived as opportunities for action offered by the environment to an organism (graspability, sit-on-ability, etc.). According to Gibson and contemporary ecological psychologists, affordances are relational properties: they are derived from the ecological relationship of interdependency between the behavioral and cognitive capacities of a given organism and the objective properties of its environment (Gibson 1979; 
Stavros Valenti and Gold 1991; Good 2007). This coupling is grounded in the long-term attunement proper to evolution: given the needs of a particular species, its hard-wiring is likely to have evolved in a direction that simplifies picking up the necessary information and parsing the environment in a specific way (Sanders 1997). For instance, worms are perceived as edible for birds but not for horses. The coupling between an organism and its environment is also grounded in the short-term attunement proper to situated actions: the structure of material objects creates new environmental properties that in turn provide organisms with an additional range of possible actions, for instance the "weaponability" of a branch (Schmidt 2007). To put it otherwise, the concept of affordances emphasizes the way in which the perceptual and actional system of fine-tuned organisms resonates with the properties of their environment-an environment that appears to them as a field of practice, a "taskcape" (Ingold 2001).

Most importantly, affordances are not restricted to situations coupling material objects to a given range of actions. Far from being limited to the physical features of a "body-scale" environment, affordances refer to what an organism "can do" in an environment that can be seen as an entire realm of potential action (Heft 1989). This means that the social environment also provides a rich and very elaborated set of affordances, including the mutual and reciprocal affordances that individuals represent for one another. "Sexual behavior, nurturing behavior, fighting behavior, cooperative behavior, economic behavior, political behavior-all depend on the perceiving of what another person or other persons afford, or sometimes on the misperceiving of it" (Gibson 1979: 135). Since, as Gibson put it, behavior affords behavior, social interactions do provide a whole range of affordances: aggressive behavior affords defensive reaction, gift affords cooperation, and kin in distress affords help. By analogy to the "demand character" of objects that Koffka (1935: 7) spoke of a long time ago, such as the fruit saying "eat me" or the water saying "drink me", social entities have also a "demand character": typical social actions and interactions afford specific kinds of responses. It is the "demand" aspect of dominance posture that affords submissiveness and it is the "demand" aspect of defenseless features of infants that afford tolerance and protection. Since they invite or even demand to act, social affordances differ in an important respect from traditional physical affordances: far from being only action possibilities or opportunities for action, they have a directive force and hence a deontic dimension. In other words, social affordances are "deontic affordances": not only they indicate "what I can do," but also "what I should do" (Carassa and Colombetti 2009; Dokic 2010). If we see an infant being molested by a grown-up, then we immediately "see" that we should rescue him. In short, social affordances are not only enabling but also constraining. For example, the "act of giving" calls for a certain social response, as shown in capuchin monkeys' reciprocal exchanges of food-for-grooming, also known as tit-for-tat: "you have given me food, then I should give you something in exchange" (Tiddi et al. 2011).

Thus deontic affordances have two important properties. First, they refer to observable, shared, public opportunities for perception and action. Although affordances are perceivable from different perspectives and that they might not be perceived or attended to, they do not fluctuate in function of the need of the perceiver: they are objective and shareable features of the environment (Gibson 1979: 139). In other words, even if affordances are not perceptually manifest in the way that colors and shapes are, they are nevertheless immediately and commonly recognizable by all the animal of a species or, at least, by all the members of a given community. Unlike mental states, generally characterized as internal, hidden, and unobservable properties of minds, deontic affordances are entirely out there, in the open, to be perceived. If we see, for example, a smiling person lifting a piece of bread toward someone else, we know that this person is offering food and that such offering gesture affords an attitude of gratitude. Second, the recognition of deontic affordances is quasi-immediate: a facial expression of intimidation affords the action of complying, and a threatening face affords the action of fleeing. In short, deontic affordances allow individuals, including those who are deprived of metarepresentational capacities (e.g., infants, non-human primates), to foresee what is the appropriate way to respond to such and such behavior without resorting to explicit norms.

Examples of deontic affordances in the non-human social world are numerous. For instance, chimpanzees facing request gestures from conspecifics are prone to share food, showing thereby that the behaviors that are recognized as requests afford or elicit pro-social responses (Yamamoto et al. 2009; Melis et al. 2011). Along the same lines, monkeys exhibit soliciting gestures that signal to the potential groomed that they want to start a grooming interaction (Matsuzawa 2008). It is worth noting that sophisticated deontic affordances are not only present in primates; indeed, in coyotes, wolves and dogs, play signals such as play bow or self-handicapping afford non-aggressive action and inhibit "serious" actions such as biting or scratching (Bateson 1972; Bekoff and Beyers 1998).

Thanks to these examples of deontic affordances, it becomes easier to see how it is possible to have "primitive normativity" in the animal and human realms. Such normativity is constitutive of the chain of actions and reactions that makes certain behaviors not only possible but also appropriate: the action of A solicits a certain response that $\mathrm{B}$, in turn, feels obligated to favor. These mutual 
solicitations, proper to social life, do not need a complex cognitive processing to be recognized and acted upon. Since action possibilities that are appropriate to enact are perceivable, their processing do not require meta-representational abilities. Just as individuals perceive that fruits make eating possible, they might readily perceive the deontic requirements that specific situations impose on behavior. ${ }^{4}$ In other words, the account of primitive normativity in terms of affordances that we defend here assumes that normative demands on action are not rules of conduct per se and, therefore, do not require a rule-following behavior whose presence in non-human societies as well as in early childhood is very unlikely. Normative demands are inherent in the course of interactions and are graspable under the form of what is the right, appropriate thing to do in such or such "affording" situation.

\subsection{From Social Affordances to Naïve Sociology}

We have seen above that an essential characteristic of social affordances is that they encapsulate a deontic force: they have a normative component that incites those who participate in a concrete interaction to act in a certain way. We will now argue that this normative aspect of concrete social interactions is the basis for a full-fledged naïve sociology, that is, a theory-like system that takes social relationships as input and forms expectations and inferences on their basis. Two aspects need to be specified for establishing how individuals can recruit such system of social inferences, even when they are not personally involved in a given interaction. Firstly, we have to insist on the fact that affordances are not only egocentric (i.e., a given feature of the environment affords the agent to act in a certain way) but also, if not mostly, allocentric (i.e., an observed social interaction enables an external observer to fore-see what will or should happen next). Secondly, we have to explain how expectancies based on the perception of concrete social interactions can be represented at a more abstract level, thereby allowing perceivers to draw inferences about the nature of a social relationship even in the absence of the main characters.

Arguably, affordances have been mostly thought of in the first person, delimiting what $I$ perceive in my environment as opportunities or calls for actions, including the potential actions of others present in my own space and hence within my reach (Dezecache et al. 2013). But it is important to note that the inviting character of affordances

\footnotetext{
${ }^{4}$ Although not resorting to the concept of affordance, several authors have argued arguing in favor of the perceptibility of deontic facts. For them, moral evaluation is not based upon conformity with rules of conduct, but upon sensitivity to deontic requirements that specific situations impose on behavior. See notably Mac Dowell (1978) and Goldie (2007).
}

can also be seen from the outside, that is, from the thirdperson point of view of a detached observer. Indeed, several researchers, including Gibson himself, have rightly pointed out that we do not only perceive affordances egocentrically; we can also see affordances allocentrically by focusing on another agent's practical abilities, opportunities and inclinations (Zaehle et al. 2006; Dokic 2010). From a cognitive point of view, allocentric perception of affordances is slightly more complex. Indeed, when we perceive an affordance from a first-person perspective (e.g., the reachability of an apple), our self does not have to be a component of our visual field. On the other hand, in the case of allocentric perception of affordances, we need to represent explicitly the relevant agent as well as his relationship with his environment-including the other individuals he is interacting with (Dokic 2010). Let us take the example of a social perceiver facing an ongoing interaction between two people, say Marc and Maurice; thanks to asymmetry display and status signaling, the perceiver detects a relational asymmetry that leads him to expect congruent conducts; he or she would be very surprised, for instance, to see Marc, who seems to be the boss, showing ostensive marks of respect (e.g., bowing, kneeling, etc.) for Maurice.

Interestingly, such allocentric perception of deontic affordances is also present in non-human primates. For instance, when a juvenile vervet monkey's scream is played from a concealed loudspeaker to three adult females, two of them look at the mother and seem to expect her to respond (Cheney and Seyfarth 1990). In other words, the adult females see the juvenile's scream as affording a caring behavior from his mother (deontic affordance) and expect the mother to act accordingly. The perspectival endorsement that affordances involve is thus far from restricted to the egocentric perception of objects; on the contrary, it includes the allocentric perception of social interactions in which others are "caught in".

Once seen the extended perspectival endorsement proper to affordances, it remains to determine which kind of supplementary ability is needed to generalize judgments of relationships, established on the basis of the affordances available in a given interaction, to another course of (inter)action. This supplementary ability is precisely what naïve sociology, as a naïve theory, is about: a system of inferences that enable social agents to extract abstract relational features from ongoing interactions and to organize them into core knowledge applicable to novel situations. In Marc and Maurice's example, observers can recruit what they know about the relationship between Marc and Maurice to infer what they should do in a new situation (e.g., making a decision, sharing food, taking the lead in an expedition, etc.). As complex as it may seem, the premises of such inferential ability exist in non-human primates. Indeed monkeys are able to generalize 
categorical judgments of dominance, inferred from the observation of a kind of interaction between unknown same-looking conspecifics (e.g., a monkey chasing another), to another kind of interaction (e.g., monkeys fighting) (Bovet and Washburn 2003). Monkeys are also able to redirect aggression against a close relative of a prior opponent, revealing a capacity for abstracting an underlying kinship relationship from a series of third-party interactions in which they are not personally involved (Cheney and Seyfarth 1990). Other studies show that chimpanzees draw inferences about the nature of an interaction they could not see: they react strongly when they hear, without any visual contact, a subordinate producing aggressor screams towards a dominant, showing thereby that they have abstract relationship-based expectancies (Slocombe et al. 2010). Deontic inferences are also generated by the relationship of group membership. For instance, members of a troop of rhesus monkeys are expected to utter the call to share food and those who do not comply are punished (Hauser and Marler 1993). On the other hand, non-members of the troop are not punished if they do not utter the call for food, revealing deontic expectations such as "if you are a member of my group, all else being equal, then I'll cooperate with you and I'll expect the same from you" (Jackendoff 1992, 1999). This kind of relational expectations does involve deontic inferences. Indeed, as Mercier and Sperber (2009) rightly remind it, an expectation, is "the outcome of an inference", typically drawn in an unconscious manner. From an evolutionary perspective, this inferential capacity is an important cognitive step that brings adaptive advantages: it enables agents to reason on the abstract level of social relationships and hence to go "beyond the information given" (Bruner and Anglin 1974).

The two-layer processing involved in naïve sociology can thus be laid out as follows:
To get back to Hinde's distinction, deontic affordances manifest the demanding character of this specific interaction (e.g., the dominant $\mathrm{x}$ requires food from subordinate $\mathrm{y}$, then $\mathrm{y}$ complies) whereas more abstract deontic principles would govern the types of social relationships (e.g., if $\mathrm{x}$ is in a relation of dominance, then $\mathrm{x}$ should perform actions $\mathrm{a}, \mathrm{b}, \mathrm{c}$, etc.). It is at this abstract level of social relationships that naïve sociology comes into play: it enables agents to go beyond the recognition of deontic affordances available in the here and now of a concrete, singular chain of interactions and to recruit more abstract deontic expectations to generate predictions about new situations. In other words, social relationships are the conceptual primitives of naïve sociology; as for the core inferential principle that supports reasoning about them, it is, from both egocentric and allocentric perspectives, of a deontic nature. Such deontic principles lead social agents to expect that two individuals engaged in a certain type of relationship at time $\mathrm{t}_{\mathrm{n}}$ should and will act accordingly at time $t_{n+1}$. For instance, two people engaged in a friendly relationship at $t_{0}$ will be expected to share food or help each other at $t_{1}$. If the course of interactions unfolded in a different way, observers would be very surprised or, at least, they would show greater orientation responses, as is the case with chimpanzees confronted to situations where rules of directionality are violated and signals are not congruent with existing social relations (Slocombe et al. 2010). For humans, one can even suppose that it is precisely in such expectation violation situations that theory of mind is preferentially activated. A psychological inquiry into the hidden beliefs or intentions of the social partner who did not behave the way he was supposed to seems indeed necessary to pinpoint the individual-level reasons for this puzzling relational discrepancy (Clément et al. 2011).

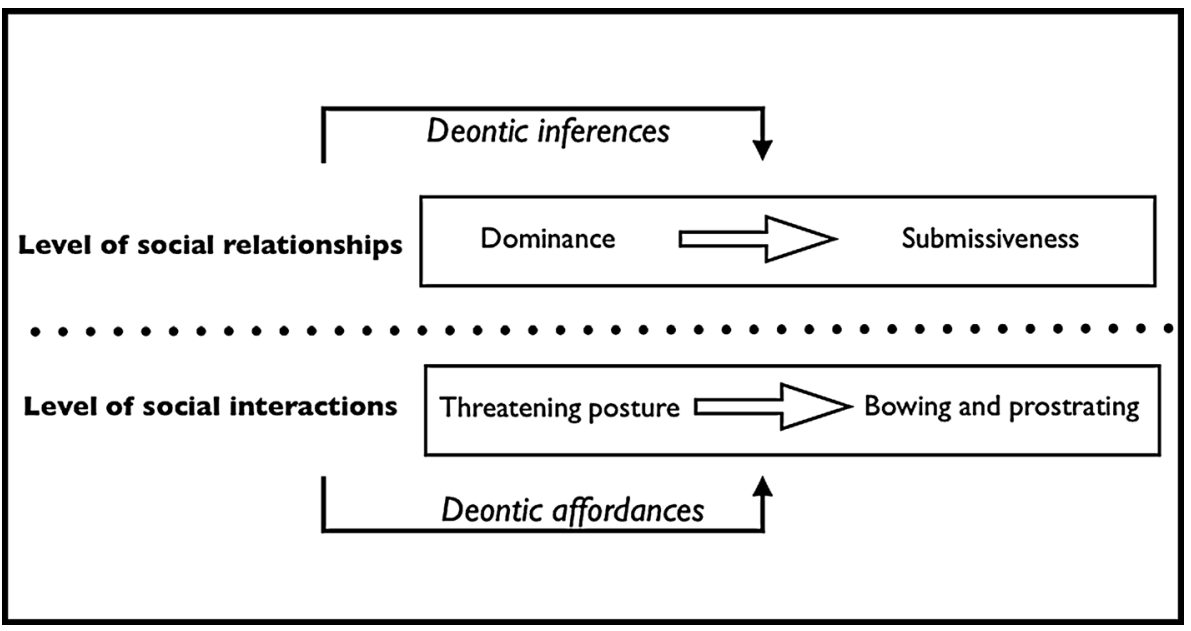




\section{From Social to Cultural Relationships}

One issue raised by our naïve sociology hypothesis concerns its application for human societies. Although one can easily admit that elementary types of social relationships do play a central role in non-human societies, one might question their relevance for human-specific and hence cultural forms of life. If, as Bloch (2008) put it, the very foundation of the sociality of humans is the capacity to imagine a "transcendental network" beyond their "immediate social circle", including the dead, ancestors and gods, the way such extended sociality can be processed by naïve sociology seems rather mysterious. Indeed our affordancebased model of naïve sociology starts from the saliency of information laid out in the environment, making it seemingly difficult to account for the "detached representations", proper to culture, which stand for objects or events that are neither present in the situation nor triggered by some recent situation (Gärdenfors 1996). In other words, since cultural representations deal mostly with things that are non perceptible, if not non-existent-whether absent people, future or hypothetical events, fictional entities or counterfactual goals (Harris 2000), what might be the usefulness of a cognitive processing ultimately grounded in the perceivable demanding character of interactions?

To answer this important question, the relational model theory proposed by Fiske $(1992,2005)$ is again useful: to him, basic social relationships take culture-specific forms of implementation and realization that children and adults coming into a new culture have to recognize and learn in order to behave in a culturally appropriate manner. If we redescribe this proposal within our framework, this means that basic social relationships constitute a core system over which each particular culture builds its own flesh: each cultural relationship would ultimately instantiate, including with supernatural beings, a basic social relationship, whether it be dominance, group membership, nurturance, cooperation, competition, or exchange.

It is worth noting that assuming that cultural types of relationships ultimately "piggyback" on basic types of social relationships does not diminish the intrinsic force of culture. By definition, culture creates new opportunities and invitations to act, but this creativity does not necessarily go against the basic social relationship repertoire that human and non-human primates would have at their disposal. This is what the following pages are going to argue about.

\subsection{Cultural "Piggybacks"}

As seen above, deontic inferences proper to primitive social relationships, as well as the deontic affordances that the instantiations of those relationships in concrete, real- life social interactions give rise to, are within the cognitive reach of young infants and non-human primates. Whereas the parsing and inferential capacities that enable social beings to "see" a relationship "as" dominance, group allegiance or exchange are shared by humans and other animal species, abilities necessary for processing cultural relationships seem to be human-specific. Indeed, the processing of cultural relationships requires species-specific symbolic abilities. Such symbolic abilities are necessary for endowing, through a series of "counting-as", natural or social facts (object, person, relationship) with new deontic, culture-specific properties (Searle 1995). Status-function assignments like "this river counts as a border", "this military salute counts as an act of submissiveness", or "this man counts as the president of the US", creates the very possibility of new kinds of activities, roles and relationships (e.g., marriage, economical exchange, political election, etc.) (Searle 2010).

This being so, although cultural types of relationships do result from a collective imaginative leap, they still might "piggyback" onto universal types of social relationships. If it were not the case, an anthropologist who does not know anything about the local cultural habits of the community she wants to investigate would be completely lost. Now, far from being lost, anthropologists demonstrate in their work that they recognize a large amount of relational commonalities in the field, as exotic as this latter may seem. In order to understand the Kula system, for instance, the wellknown anthropologist Bronislaw Malinowski focused first on familiar social relationships such as economic transaction, sense of reciprocity and hierarchical respect (Malinowski 1922). In other words, his attention was drawn to the strangeness of some conducts because those latter stood out from numerous recognizable, easily processed social relationships. To take another example from the classical anthropological literature, what makes the institution of the gift understandable is the relationship of reciprocal exchange and mutual obligation that underlies it (Mauss 1968). In spite of its cultural sophistications, gift giving and the social bond it contributes to solidify are not completely human specific; after all, the cultural ritual of giving gifts affords reciprocated countergifts just as grooming affords reciprocating in non-human primate forms of life.

Another good example of a continuum between the social and the cultural is a deontic affordance such as request. Indeed non-human primates use, in a dyadic way, request gestures to obtain something that they want from others, like grooming, play or mating, and continue to gesture until they finally receive the appropriate response (Liebal and Call 2012). Request gestures thus provide deontic affordances that are meant to trigger others into action. Although requests take more sophisticated forms in human communication, they serve the same purpose: 
providing, as Carassa and Colombetti (2009) put it, a wide range of deontic affordances that "bring about effects in others". For instance, Carassa and Colombetti say, a man asking a lady whether she would like to have a drink with him creates a deontic affordance for her, in this case the opportunity for her to accept the invitation and hence to spend some time with him. In both human and non-human communication, requests provide affordances for others to act in a certain way.

Even abstract cultural phenomena can be integrated into a naturalist continuum that goes from the basic requirements of living in social groups to the specific demands and rules for action of the culture we live in. So a nation, even if it involves an imaginative leap that only humans are capable of, can be seen as a very sophisticated sociocultural elaboration of the basic relationship of group membership. Of course, one must not diminish the difference between experience-based group membership and imaginative, nation-like group membership. A "knowable community", constituted and maintained through face-to-face interactions, greatly differs from the "imagined community" that comes only into existence thanks to the certainty, in the mind of each of its members, that they do form a community (Anderson 1983). Still, in spite of this important change of scale, group membership works similarly enough in these two kinds of communities to trigger the same deontic inferences, proper to naïve sociology. For instance, just like primates expect cooperation and coalitionary behavior from in-group members in case of intergroup conflict (Muller and Mitani 2005), people who identify others as being part of their national community expect them to be on their side in case of international conflict.

Although requiring an important imaginative stretch, complex systems of cultural beliefs are also grounded in more basic types of social relationships. For instance, the anthropologist Favret-Saada (1977, 2009) has forcefully demonstrated that, in the France of the 1970s, the stake of witchcraft was nothing but the attempt, often cruel, to reestablish the fundamental social equilibrium jeopardized by the alleged witch. The witch was seen as a free rider, who got rich at the expense of others and malevolently transgressed the elementary rules of social exchange and reciprocation necessary to hold society together (FavretSaada 1977, 2009; Clément 2003). Among numerous other studies, this research shows that an important part of culture ultimately refers to high-level, culture-dependent redescriptions and reconfigurations of primitive types of social relationships. The processing of this cultural repertoire, we suggest, is facilitated by the mastery of a mature naive sociology that enables the understanding of complex deontic systems of relationships such as commercial transaction, political election, marital engagement, or public instruction. An important function of these rights and duties systems is indeed to establish the systems of interdependent places (e.g., seller-buyer, president-elector, husband-wife, teacher-student) that define the scope of what can be uttered and done by whom in which situation. So when the teacher speaks, students must remain silent; when I marry you, then I have to take care of you, and so on. Interestingly, deontic principles at the core of basic social relationships work in the same way: they specify how role "bearers" such as superior-inferior or motherchild ought to relate to one another.

Even if social and cultural relationships have a different degree of complexity and do not require the same cognitive capacities to be processed, it is important to note that this does not call into question the hypothesis of naïve sociology. Mature naïve sociology does not work differently than mature naïve psychology. Indeed mature naïve psychology involves successive and diverse conceptual acquisitions, from basic desire understanding to complex understanding of the divine will of God (Wellman 1998). Similarly, mature naïve sociology allows the processing of more or less complex types of relationships, from rudimentary affiliative and dominance relationships to institutional division of labor. The fact that the wide range of cultural relationships involves distinct emotional and motivational constituents and different developmental processes does not turn for all that mature naïve sociology into a conceptual umbrella for a variety of disparate, unrelated concepts of relationships. Just like naïve psychology, naïve sociology might be a developmental construct that broadens with ontogeny and socialization but is nevertheless grounded in a rather limited conceptual repertoire of social relationships. Cognitive competences necessary to process cultural relationships and to pick out the deontic affordances that instantiate them in situ derive from those enabling human and non-human primates to process their social counterpart.

\subsection{The Cultural Shaping of Affordances}

If cultural relationships are built up on universal types of social relationships, common to all human societies, it remains to explain how cultural differences can emerge and be maintained. The hypothesis that we would like to defend here is that cultural differences emerge from the uneven degree of attention granted to the social relationships that carve the social world in a universal manner.

The salience of social characteristics, such as gender, race, ethnicity, age, cultural membership, and social status, varies from culture to culture (Freeman et al. 2009). A large amount of evidence thus shows cultural variation in the degree of salience of relationships and of their situated instantiation in social affordances. In Japan, for instance, 
there is more affordance than in the United State to be subordinate, as subordinate thinking and behavior is positively reinforced (Moskowitz et al. 1994). Culture can reinforce or inhibit the deontic force of social but also physical affordances, giving individuals the incentive to recognize some affordances as worthy of being acted upon or, on the contrary, as being irrelevant or meaningless. The attentional paths that thus implicitly restrain or extend the individuals' scope of possible actions, perceptions, and representations are at the heart of the process of enculturation. Such process is primarily performed by the "attention-directing interactions" of attention holders (e.g., caregivers, teachers, authority figures, and so on) (Loveland 1991: 110). To become a competent community member, we have to learn the attentional commonalities that mediate the perception, qualification and recognition of saliencies, define what information is relevant in which situation, and inhibit or encourage opportunities for action. Whereas social affordances such as threatening faces or request gestures can be immediately recognized, cultural affordances require "training" in a given cultural environment to be recognized and acted upon. In other words, community members must acquire the sense of possibilities, incentives, and obligations to act that characterize their culture and learn to identify them in the layout of their environment (e.g., deference to authority figures, meat taboos, dress codes, etc.). For instance, in England, they have to learn that the queen entering a room affords a given response, in this case standing up; they must more generally learn that this deontic affordance instantiates the abstract cultural relationship of respect and subordination that relates the people to its queen. In the same vein, the inhabitants of a lot of countries must learn that the flag is a cultural affordance that affords saluting it (Windsor 2004) because it instantiates the abstract cultural relationship of nation membership.

Several studies in ecological psychology, cognitive sociology and social psychology lend support to the hypothesis that cultural differences might emerge first from the fine-grained socialization or enculturation of attention. The culture-dependency of the "attentional bias" that leads agents to selectively attend to the relevant features of their physical and social environments is strikingly revealed in cross-cultural and cross-social experimental studies on logical inference, perception, categorization and causal analysis (Nisbett et al. 2001; Nisbett 2003). For instance, Easterners give priority to relationship processing, background elements and holistic explanations, whereas Westerners focus on individual properties, mentalistic attributions and analytic thinking (Nisbett 2003). Because of these cultural 'lenses', even a simple scene of fish swimming in an aquarium is perceived differently: Westerners are inclined to focus on the substantial, intrinsic properties of the fish themselves (e.g., color, shape, etc.), while East Asians tend to pay attention to the field and to the relations between the fish and the field.

Interestingly, those different attentional pathways seem to be explained by the specificity of the social organization and relational form of life people live in. Indeed, farming and fishing communities that emphasize harmonious social interdependence exhibit greater holistic cognitive tendencies in attention, categorization, and reasoning than herding community-even if the three types of communities belong to the same national, geographic, ethnic, and linguistic regions, in this case the region of Turkey's eastern Black Sea (Uskul et al. 2008). Similarly, interdependent southern Italians and working class people, who prompt attention to social relationships, reason in a more holistic fashion than relatively independent northern Italians or middle class individuals (Knight and Nisbett 2007). These different studies show that what is "seen as" relevant information depends on the way societies are organized and orient attention toward some aspects of the environment at the expense of others.

This attention-directing work is particularly manifest in the variation of cultural patterns of attention and interaction. Comparing the interactions of Japanese and American mothers with their 6, 12 and 19 months old children, Fernald and Morikawa (1993) have thus shown that American mothers use twice as many "object labels" (e.g., doggie, piggie) as Japanese mothers, whereas the latter use twice as many "social routines of politeness forms" (empathy, greeting). For Nisbett (2003), these results suggest that interactional style as well as beliefs about child rearing strongly influence the structure and content of speech addressed to infants and, above all, triggers culture-specific patterns of attention. Whereas American children are learning to be especially attentive to the place of objects, Japanese children are learning that relationships are the most relevant aspects of the world (Nisbett 2003).

Focusing on the attention-directing interactions of caregivers and the attentional pathways that the physical, social and cultural environment may afford or elicit makes cultural variations less mysterious. Certain features of the environment, as well as certain cognitive or emotional experiences, are the constant focus of cultural attention and are, as Levy (1973) put it, "hypercognized"; others are, on the contrary, "hypocognized", that is, virtually ignored. For instance, in an American culture characterized by its "allergy to the past", regret is considered as an unhealthy, unproductive backward-looking emotion and is "hypocognized" to the point of disappearing from the phenomenological mindscape of American people (Landman 1996). Just as "inattentional blindness" can occur, at a perceptual level, by focused attention on some details at the expense of others, even if 'this detail' is a gorilla (Simons and Chabris 
1999), inattentional blindness can also occur at other cognitive levels. Attention and disattention are thus the main workings of the enculturation process that teaches us to be "affected" by specific deontic affordances. As Zerubavel (1997) suggests, individuals must learn the topology of salience of their cultural community, whether it be the beauty of a landscape, the similarity of sexes instead of their differences, or the relevance of the lions in the zoo instead of the fences. In this "optical socialization", the deontic component is essential: the optical or attentional deviants who are at odds with the saliencies commonly shared by others around them and do not respond to public, common affordances risk social "excommunication".

\section{Conclusion: Intertwining Social and Natural Sciences}

At a time when even biologists insist on the importance of the social environment for understanding the evolution of the human brain, any emphasis on an incompressible divide between cognitive science and social science seems outdated. To show social scientists that cognitive sciences do not obligatory involve a reduction of their traditional areas of interest to mental processes, we have focused our argument on the existence of an independent cognitive domain dedicated to the processing of social relationships: naïve sociology.

One of the interests of our naïve sociology hypothesis is that it extends the way most cognitive scientists frame social cognition. Indeed, the prevailing view of the social domain consists, from an ontological point of view, of mental states and, from a cognitive point of view, of mindreading. This view of social cognition, we argue, needs to be supplemented with another conception of the social domain, characterized by a relational ontology and responding to the deontic logic of social relationships. Social relationships have a directive force: social beings feel in principle committed to them, regard themselves as obligated to abide by them, and impose them on conspecifics-including third parties (Fiske 1992, 2005; Haslam and Fiske 2004). So deontic inferences specific to naïve sociology, we claim, do not depend on theory of mind: they constitute a cognitive domain of their own. In its elementary and universal manifestation, mostly shared with nonhuman primates, as well as in its mature and cultural reconfiguration, naïve sociology allows well-adapted social beings to grasp the constraining and enabling affordances that are laid out in the common world 'out there' (deontic affordances). Deontic affordances are not mere action possibilities or opportunities for action but solicitations to act. The temporal scale and cognitive impact of those solicitations can vary since they can be either contingent on the situated interaction (Carassa and Colombetti 2009), dependent upon the socio-historical context, or more permanent and rooted in our evolutionary history (Withagen et al. 2012). But in any cases deontic affordances can be perceived either egocentrically for oneself or allocentrically for other, encompassing thereby both the responsive abilities of the social agent and the interpretative skills of the social perceiver. Since they indicate what individuals should do in which situation, deontic affordances are easily translatable into the more generic deontic principles that are at the core of naïve sociology. They enable social agents to infer, on their basis, the abstract patterns of relationships that govern them (deontic inferences). To sum up, naïve sociology enables anyone, but in particular newcomers to a social group such as children or immigrants, to rapidly identify social and cultural relationships as well as the deontic affordances that characterize their instantiation in concrete chains of interactions.

Another interest of our naïve sociology hypothesis is that it involves a model of the mind that has plausible correlates in the mental processes described by psychologists and cognitive scientists. Currently, indeed, the metatheoretical assumptions on the social nature of the human mind that underlie the prevailing approaches in the social sciences are rarely transformed into an empirical enquiry. Such empirical investigation can force social theorists to be, at last, realistic about the mental (Kaufmann 2011). The human mind, even if it requires a cultural scaffolding to participate in ordinary social life, is far from the "blank slate" that most models of the social sciences take for granted (Pinker 2002). The mind is endowed with a cognitive equipment, socially "in-formed", that demonstrates that mind and society are not inversely proportional. Scientists, whether they be cognitive or social scientists, do not have to choose between the internal foundations and the external features of the social. A social behavior is by definition the joint co-product of cognitive and social processes. As the philosopher Dretske (1988) put it, the behavior of an individual is provoked by a "triggering cause", necessarily proximate and cognitively instantiated, which is immediately responsible for its occurrence (e.g., Clyde stood up because he saw the queen enter the room). If cognitive scientists can pinpoint what causes this particular individual to do $x$ rather than $y$, they still need the help of social scientists to describe the "structuring causes", that is, the distal phenomena that have shaped or structured the behavioral process as a whole (e.g., Clyde stood up as a gesture of respect) (Dretske 1988: 43-45). As far as acculturated animals like humans are concerned, many of these structuring causes are extrinsic to the individual and social in nature. We have suggested that those social causes can influence individual behaviors through the numerous deontic affordances to which our cognitive system is particularly sensitive. 
Of course, there is much work to be done in the future to better specify naïve sociology. First of all, further research is needed to identify the different kinds of social relationships that furnish the human and non-human worlds. In order to do so, a cognitive approach, focused on the identification of the internal processes that enable us to adjust to the social world, needs to be supplemented with a sociological approach, centered on the external description of canonical social and cultural relationships. Such a sociological approach would greatly benefit from observational techniques of ethologists, which combine naturalist concern and descriptive sharpness (Altmann 1974; Zuberbühler and Wittig 2011). In parallel, standard experimental methodologies developed by cognitive scientists are needed to understand how social relationships and deontic affordances might be cognitively recognized and recruited. One important issue would be to highlight, mainly thanks to developmental psychology, the relationship between naïve sociology and naïve psychology, as they would be the two main domains of social cognition. More and more studies seem to indicate that young infants develop expectancies about the behaviors of people involved in specific kinds of relationship such as dominance (Mascaro and Csibra 2012). But it still remains to find the "acid test" that would prove that deontic affordances and inferences proper to naïve sociology take a cognitive pathway different from that of naïve psychology.

Last but not least, we have argued that culture comes mostly to the mind in the form of the attentional paths that implicitly restrain or extend the scope of possible actions and perceptions. We have also argued that social and cultural relationships can be integrated into a naturalist continuum that indicate what can and should be done. But both arguments are underlain by an important distinction, remained implicit until now, which needs to be highlighted: the distinction, too often left aside in the social sciences, between the social and the cultural. Even if the social and the cultural might be difficult to pull apart in empirical, particular social situations, their distinction must be analytically maintained because they do not have the same phylogenetic, and probably ontogenetic, history. Whereas elementary forms of sociality are found in nonhuman societies, cultural relationships are artificial, "linguistically infected" and therefore human-specific. Culture presupposes the existence of the social, but the reverse is not true: a culture consists of shared representations and practices whose maintenance through time requires preexisting social relationships that structure their circulation and transmission (Kroeber and Parsons 1958; Kaufmann and Clément 2003; Schaeffer 2007). In other words, cultural relationships are grounded in social relationships but it does not work the other way around.
This does not mean that culture does not have fundamental implications for social life, even when it refers to symbolic, non-perceptible entities. Indeed, at first glance, symbolic representations such as the Holy Trinity seem difficult to apprehend within a naturalist framework. Separated by a huge gap from basic social forms of life, they are neither reducible to the oriented screening of preexisting social affordances, nor to the creation of new readily perceptible opportunities for action. Yet symbolic representations have a deontic force that enables them to operate as 'social glue'. The "contractual intangibles" proper to culture, such as spirits, divinities, laws or nations, have a strong impact on social integration and cooperation by promoting moral cohesion and collective allegiance (Dunbar et al. 1999).

In other words, symbolic representations have a "togetherness effect": they trigger a participative stance that allows individuals to go beyond their own narrow sphere of experience to better reach the sphere of the collective imagination. This participative stance leads the individuals to enter an imaginary world that provides them with new opportunities of action and can, in fine, transform their existence. However, such a stance does not override the natural inclinations of our basic social mind: it rather extends them to cultural entities whose deontic force needs to be highlighted by both social and cognitive sciences.

Acknowledgments The authors are grateful to Hugo Mercier for his fruitful remarks on the manuscript. We also thank an anonymous reviewer for his/her very helpful suggestions and criticisms.

\section{References}

Altmann J (1974) Observational study of behavior: sampling methods. Behaviour 49:227-267

Anderson B (1983) Imagined communities. Verso, London

Andrews K (2009) Understanding norms without a theory of mind. Inquiry 52(5):433-448

Astington JW (2004) Bridging the gap between theory of mind and moral reasoning. New Dir Child Adolesc Dev 103:63-72

Atran S (1998) Folk biology and the anthropology of science: cognitive universals and cultural particulars. Behav Brain Sci 21(4):547-609

Baillargeon R (1987) Object permanence in 31/2 and 41/2-months-old children. Dev Psychol 23:655-664

Baillargeon R, Carey S (2012) Core cognition and beyond: the acquisition of physical and numerical knowledge. In: Pauen S (ed) Early childhood development and later outcome. Cambridge University Press, London, pp 33-65

Baron-Cohen S, Tager-Flusberg H, Cohen DJ (eds) (2000) Understanding other minds. Perspectives from autism. Oxford University Press, Oxford

Bateson G (1972) Steps to an ecology of mind. Chandler, San Francisco

Beier JS, Carpenter M, Tomasello M (2010) Young children's understanding of third-party social relationships. In: Poster 
presented at the XVIIth biennial international conference on infant studies, Baltimore

Bekoff M, Beyers JA (1998) Intentional communication and social play: how and why animals negotiate and agree to play. Cambridge University Press, Cambridge

Bloch M (2008) Why religion is nothing special but is central. Philos Trans R Soc B 363(1499):2055-2061

Bourdieu P (1979) La distinction. Minuit, Paris

Bovet D, Washburn DA (2003) Rhesus macaques (macaca mulatta) categorize unknown conspecifics according to their dominance relations. J Comp Psychol 117(4):400-405

Bronner G (2006) L'acteur social est-il (déjà) soluble dans les neurosciences ? L'Année sociologique 56(2):331-352

Bruner JS, Anglin JM (1974) Beyond the information given: studies in the psychology of knowing. G. Allen and Unwin, London

Carassa A, Colombetti M (2009) Joint meaning. J Pragmat 41(9): 1837-1854

Carey SE, Spelke E (1996) Science and core knowledge. Philos Sci 63(4):515-533

Charafeddine R, Mercier H, Clément F, Kaufmann L, Reboul A, Van der Henst J-B (submitted) Children's ability to recognize dominance and attitudes towards dominant individuals

Cheney DL, Seyfarth RMS (1990) How monkeys see the world, inside the mind of another species. University of Chicago Press, Chicago

Cheney DL, Seyfarth RMS (2007) Baboon metaphysics. The evolution of a social mind. Chicago University Press, Chicago

Clément F (2003) L'esprit ensorcelé. Les racines cognitives de la sorcellerie, Terrain 41:121-136

Clément F, Bernard S, Kaufmann L (2011) Social cognition is not reducible to theory of mind. When children use deontic rules to predict others' behaviors. Br J Dev Psychol 29(4):910-928

Cosmides L, Tooby J (1994) Origins of domain specificity: the evolution of functional organization. In: Hirschfeld LA, Gelman SA (eds) Mapping the mind. Domain specificity in cognition and culture. Cambridge University Press, Cambridge, pp 85-116

Cosmides L, Tooby J, Kurzban R (2003) Perceptions of race. Trends Cogn Sci 7(4):173-179

Cosmides L, Tooby J, Fiddick L, Bryant GA (2005) Detecting cheaters. Trends Cogn Sci 9(11):505-551

Cummins DD (1999) Cheater detection is modified by social rank: the impact of dominance on the evolution of cognitive functions. Evol Hum Behav 20(4):229-248

Dehaene S, Spelke E, Pinel P, Stanescu R, Tsivkin S (1999) Sources of mathematical thinking: behavioral and brain-imaging evidence. Science 284:970

Dennett D (1996) Kinds of mind. Basic Books, New York

Descombes V (1996) Les institutions du sens. Minuit, Paris

Dezecache G, Conty L, Grèzes J (2013) Social affordances: is the mirror neuron system involved? Commentary on target article of Schilbach and colleagues. Behav Brain Sci 36(4):417-418

Diesendruck G, Eldror E (2011) What children infer from social categories. Cogn Dev 26(2):118-126

Dilthey W (1883/1999) Critique de la raison historique: introduction aux sciences de l'esprit et autres textes. Cerf, Paris

Dokic J (2010) Affordances and the sense of joint agency. In: Balconi $M$ (ed) Neuropsychology of the sense of agency. Springer, Milan, pp 23-43

Dretske F (1988) Explaining behavior: reasons in a world of causes. MIT Press, Cambridge, MA

Dunbar RIM (2003) Evolution of the social brain. Science 302(5648):1160-1161

Dunbar R, Knight C, Power C (eds) (1999) The evolution of culture: an interdisciplinary view. Edinburgh University Press, Edinburgh, pp 1-11
Dunham Y, Baron AS, Banaji MR (2008) The development of implicit intergroup cognition. Trends Cogn Sci 12:248-253

Durkheim E (1991) [1912] Les formes élémentaires de la vie religieuse. Librairie Générale Française, Paris

Favret-Saada J (1977) Les Mots, la mort, les sorts: la sorcellerie dans le bocage. Gallimard, Paris

Favret-Saada J (2009) Désorceler. L'Olivier, Paris

Fernald A, Morikawa H (1993) Common themes and cultural variations in Japanese and American mothers' speech to infants. Child Dev 64:637-656

Fiske AP (1992) The four elementary forms of sociality: framework for a unified theory of social relations. Psychol Rev 99(4):689-723

Fiske AP (2005) Social relations: culture, development, natural selection, cognition, the brain, and pathology. In: Van Lange PAM (ed) Bridging social psychology: the benefits of transdisciplinary approaches. Erlbaum, Mahwah, pp 293-300

Fiske AP, Fiske ST (2007) Social relationships in our species and cultures. In: Kitayama S, Cohen D (eds) Handbook of cultural psychology, Guilford, New York, pp 283-306

Flack JC, Jeannotte LA, de Waal FBM (2004) Play signaling and the perception of social rules by juvenile chimpanzees (pan troglodytes). J Comp Psychol 118(2):149-150

Freeman JB, Rule NO, Adams RB, Ambady N (2009) Culture shapes a mesolimbic response to signals of dominance and subordination that associates with behavior. Neuroimage 47:353-359

Gärdenfors P (1996) Cued and detached representations in animal cognition. Behav Process 36:263-273

Garfinkel H (1967) Studies in ethnomethodology. Prentice-Hall, Englewood Cliffs, NJ

Gelman R, Williams EM (1998) Enabling constraints for cognitive development and learning: domain specificity and epigenesis. In: Kuhn D, Siegler RS (eds) Handbook of child psychology, vol II, 5th edn. Wiley, New York, pp 575-630

Gibson J (1979) The ecological approach to visual perception. Houghton Mifflin, Boston

Ginsborg H (2011) Primitive normativity and skepticism about rules. J Philos 108(5):227-254

Goldie P (2007) Seeing what is the kind thing to do: perception and emotion in morality. Dialectica 61(3):347-361

Good JM (2007) The affordances for social psychology of the ecological approach to social knowing. Theory Psychol 17:265-295

Grosenick L, Clement TS, Fernald RD (2007) Fish can infer social rank by observation alone. Nature 445:429-432

Hacking I (1999) The social construction of what? Harvard University Press, Cambridge Mass

Harris PL (2000) The work of the imagination. Blackwell, Oxford

Harris PL (2006) Social cognition. In: Damon W, Lerner RM, Kuhn D, Siegler R (eds) Handbook of child psychology, vol 2, 6th edn. New York, Wiley, pp 811-856

Haslam N (ed) (2004) Relational models theory: a contemporary overview. Lawrence Erlbaum, NJ

Haslam N, Fiske AP (2004) Social expertise: theory of mind or theory of relationships? In: Haslam N (ed) Relational models theory: a contemporary overview. Erlbaum, Mahwah, NJ, pp 147-163

Hauser MD, Marler P (1993) Food-associated calls in rhesus macaques (Macaca mulatta): II. Costs and benefits of call production and suppression. Behav Ecol 4:206-212

Havelange V (1998) Le social en débat: cognition ou interprétation. Intellectica 26-27:9-55

Hawley PH (1999) The ontogenesis of social dominance: a strategybased evolutionary perspective. Dev Rev 19:97-132

Heft H (1989) Affordances and the body: an intentional analysis of Gibson's ecological approach to visual perception. J Theory Soc Behav 19(1):1-30 
Herrmann E, Call J, Hernàndez-Lloreda MV, Hare B, Tomasello M (2007) Humans have evolved specialized skills of social cognition: the cultural intelligence hypothesis. Science 317:1360-1366

Hinde RA (1976) Interactions, relationships and social structure. Man 11:1-17

Hirschfeld LA (1995) Do children have a theory of race? Cognition 54:209-252

Hirschfeld LA (1999) Naïve sociology. In: Wilson R, Keil F (eds) The MIT encyclopedia of the cognitive sciences. MIT Press, Cambridge, MA, pp 579-580

Hirschfeld LA (2001) On a folk theory of society: children, evolution, and mental representations of social groups. Pers Soc Psychol Rev 5:107-117

Hirschfeld LA (2006) Who needs a theory of mind. In: Viale R, Andler D, Hirschfeld L (eds) Biological and cultural biases of human inference. Lawrence Erlbaum, Mahwah, NJ, pp 131-157

Hirschfeld LA, Gelman SA (1994) Mapping the mind. Domain specificity in cognition and culture. Cambridge University Press, Cambridge

Hirst P, Wooley P (1982) Social relations and human attributes. Tavistock, London

Humphrey NK (1976) The social function of intellect. In: Bateson PPG, Hinde RA (eds) Growing points in ethology. Cambridge University, Cambridge, pp 303-317

Ingold T (2001) From the transmission of representations to the education of attention. In: Whitehouse $\mathrm{H}$ (ed) The debated mind. Berg, New York, pp 113-153

Jackendoff RS (1992) Languages of the mind: essays on mental representation. MIT Press, Cambridge

Jackendoff RS (1994) Patterns in the mind. Language and human nature. Basic Books, New York

Jackendoff RS (1999) The natural logic of rights and obligations. In: Jackendoff R, Bloom P (eds) Language, logic, and concepts: essays in memory of John Macnamara. MIT Press, Cambridge, MA, pp 67-95

Jackendoff RS (2007) Language, consciousness, culture: essays on mental structure. MIT Press, Cambridge

Kaufmann L (2011) Social minds. In: Jarvie I, Zamora J (eds) The sage handbook of the philosophy of social sciences. Sage, London, pp 153-180

Kaufmann L, Clément F (2003) La sociologie est-elle un savoir infus? De la nature sociale de l'architecture cognitive. Intellectica 36(37):421-457

Kaufmann L, Cordonier L (2011). Vers un naturalisme social. Sociologies. http://sociologies.revues.org/3743

Keil F (1998) Cognitive science and the origins of thought and knowledge. In: Damon W, Lerner RM (eds) Handbook of child psychology, vol I. Wiley, New York, pp 341-413

Kinzler K, Spelke E (2011) Do infants show social preferences for people differing in race? Cognition 119(1):1-9

Knight N, Nisbett RE (2007) Culture, class and cognition: evidence from Italy. J Cogn Cult 7:283-291

Koffka K (1935) Principles of Gestalt psychology. Lund Humphries, London

Kroeber A, Parsons T (1958) The concept of culture and the social system. Am Sociol Rev 23(5):582-583

Kurzban R, Tooby J, Cosmides L (2001) Can race be erased? Coalitional computation and social categorization. Proc Natl Acad Sci USA 98:15387-15392

Landman J (1996) Social control of "negative" emotions: the case of regret. In: Harré R, Parrott WG (eds) Emotions: social, cultural and biological dimensions. Sage, London, pp 89-116

Leslie AM (1991) Theory of mind impairment in autism. In: Whiten A (ed) Natural theories of mind: evolution, development, and simulation of everyday mindreading. Basil Blackwell, Cambridge, MA, pp 63-78
Levy R (1973) Tahitians: mind and experience in the Society Islands. University of Chicago Press, Chicago

Liebal K, Call J (2012) The origins of non-human primates' manual gestures. Philos Trans R Soc B 367(1585):118-128

Loveland K (1991) Social affordances and interaction II: autism and the affordances of the human environment. Ecol Psychol 3:99-119

Mac Dowell J (1978) Are moral requirement hypothetical imperatives? Proc Aristot Soc 52:13-29

Malinowski B (1922) Argonauts of the western Pacific: an account of native enterprise and adventure in the archipelagos of Melanesian New Guinea. Routledge, London

Mascaro O, Csibra G (2012) Representation of stable social dominance relations by human infants. Proc Natl Acad Sci USA 109(18):6862-6867

Matsuzawa T (ed) (2008) Primate origins of human cognition and behavior. Springer, Tokyo

Mauss M (1968) Sociologie et anthropologie. PUF, Paris

Melis AP, Warneken F, Jensen K, Schneider A, Call J, Tomasello M (2011) Chimpanzees help conspecifics to obtain food and nonfood items. Proc R Soc Biol 278:1405-1413

Mercier H, Sperber D (2009) Intuitive and reflective inferences. In: Evans J, Frankish K (eds) In two minds: dual processes and beyond. Oxford University Press, Oxford, pp 149-170

Moskowitz DS, Suh EJ, Desaulniers J (1994) Situational influences on gender differences in agency and communion. J Pers Soc Psychol 66:753-761

Muller MN, Mitani JC (2005) Conflict and cooperation in wild chimpanzees. Adv Study Behav 35:275-331

Nisbett R (2003) The geography of thought: how Asians and westerners think differently... and why. Free Press, New York

Nisbett R, Peng K, Choi I, Norenzayan A (2001) Culture and systems of thought. Psychol Rev 108:291-310

Nucci L (2001) Education in the moral domain. Cambridge University Press, Cambridge

Ogien A (2011) Les sciences cognitives ne sont pas des sciences humaines. Sociologies. http://sociologies.revues.org/3635

Paz-y-Miño GC, Bond AB, Kamil A, Balda RP (2004) Pinyon jays use transitive inference to predict social dominance. Nature 430:778-781

Pinker S (2002) The blank slate. Viking, New York

Quéré L (2011) De vieilles obsessions sous des habits neufs? Sociologies. http://sociologies.revues.org/3744

Rhodes M (2012) Naïve theories of social groups. Child Dev 83:1900-1916

Rudolf von Rohr C, Burkart JM, van Schaik CP (2011) Evolutionary precursors of social norms in chimpanzees: a new approach. Biol Philos 26(1):1-30

Sanders JT (1997) An ontology of affordances. Ecol Psychol 9:97-112

Schaeffer J-M (2007) La fin de l'exception humaine. Gallimard, Paris

Schmidt RC (2007) Scaffolds for social meaning. Ecol Psychol 19:137-151

Searle JR (1995) The construction of social reality. The Penguin Press, London

Searle J (2010) Making the social world. The structure of human civilization. Oxford University, Oxford

Shutts K, Kinzler KD, Katz RC, Tredoux C, Spelke ES (2011) Race preferences in children: insights from South Africa. Dev Sci 14(6):1283-1291

Silk JB (1999) Male bonnet macaques use information about thirdparty rank relationships to recruit allies. Anim Behav 58:45-51

Simons DJ, Chabris CF (1999) Gorillas in our midst: sustained inattentional blindness for dynamic events. Perception 28:1059-1074

Slocombe KE, Kaller T, Call J, Zuberbühler K (2010) Chimpanzees extract social information from agonistic screams. PLoS One 5:e11473 
Spaulding S (2010) Embodied cognition and mindreading. Mind Lang $25: 119-140$

Spelke E (1994) Initial knowledge: six suggestions. Cognition 50:431-445

Spelke ES, Kinzler KD (2007) Core knowledge. Dev Sci 10(1):89-96

Spelke E, Bernier EP, Skerry AE (2013) Core social cognition. In: Mahzarin R, Banaji MR, Gelman SA (eds) Navigating the social world. What infants, children, and other species can teach us. Oxford University Press, Oxford, pp 11-16

Sperber D, Premack D, Premack AJ (1995) Causal cognition: a multidisciplinary debate. Oxford University Press, New York

Stavros Valenti S, Gold JM (1991) Social affordances and interaction I: introduction. Ecol Psychol 3:77-98

Strayer FF, Strayer J (1976) An ethological analysis of social agonism and dominance relations among preschool children. Child Dev 47(4):980-989

Sultanescu O, Andrews K (2013) Are apes' responses to pointing gestures intentional? Humana Mente J Philos Stud 24:53-77

Thomsen L, Carey S (2013) Core cognition of social relations. In: Banaji MR, Gelman SA (eds) Navigating the social world: what infants, children and other species can teach us. Oxford University Press, New York

Thomsen L, Frankenhuis WE, Ingold-Smith MC, Carey S (2011) Big and mighty: preverbal infants mentally represent social dominance. Science 331(6016):477

Tiddi B, Aureli F, Polizzi di Sorrentino E, Janson C, Schino G (2011) Grooming for tolerance? Two mechanisms of exchange in wild tufted capuchin monkeys. Behav Ecol 22:663-669

Tomasello M (1999a) The cultural origins of human cognition. Harvard University Press, Cambridge, MA

Tomasello M (1999b) The human adaptation for culture. Annu Rev Anthropol 28:509-529

Turiel E (1983) The development of social knowledge. Morality and convention. Cambridge University Press, Cambridge
Uskul A, Kitayama S, Nisbett R (2008) Ecocultural basis of cognition: farmers and fishermen are more holistic than herders. Proc Natl Acad Sci 105(25):8552-8855

Watts DP (2010) Dominance, power, and politics in nonhuman and human primates. In: Kappeler PM, Silk JB (eds) Mind the gap: tracing the origins of human universals. Springer, Berlin, pp 109-138

Weber M [1904] (1949) The methodology of the social sciences. Free Press, Glencoe

Wellman HM (1990) The child's theory of mind. MIT Press, Cambridge

Wellman HM (1998) Culture, variation, and levels of analysis in folk psychologies: comment on Lillard (1998). Psychol Bull 123(1): 33

Winch P (1958) The idea of a social science and its relation to philosophy. Routledge, London

Windsor WL (2004) An ecological approach to semiotics. J Theory Soc Behav 34(2):179-198

Withagen R, de Poel H J, Araújo D, Pepping G-J (2012) Affordances can invite behavior: reconsidering the relationship between affordances and agency. New Ideas Psychol 30(2):250-258

Yamamoto S, Humle T, Tanaka M (2009) Chimpanzees help each other upon request. PLoS One 4:e7416

Zaehle T, Jordan K, Wustenberg T, Baudewig J, Dechent P, Mast F (2006) The neural basis of the egocentric and allocentric spatial frame of reference. Brain Res 1137:92-103

Zerubavel E (1997) Social mindscapes. An invitation to cognitive sociology. Harvard University Press, Cambridge MA

Zuberbühler K, Wittig RM (2011) Field experiments with nonhuman primates: a tutorial. In: Setchell J, Curtis DJ (eds) Field and laboratory methods in primatology: a practical guide. Cambridge University Press, Cambridge, pp 207-224 